\title{
Pediatric Emergency Department Visits Before and During the COVID-19 Pandemic — United States, January 2019-January 2022
}

\begin{abstract}
Lakshmi Radhakrishnan, $\mathrm{MPH}^{1}$; Kelly Carey, $\mathrm{MPH}^{1}$; Kathleen P. Hartnett, $\mathrm{PhD}^{1}$; Aaron Kite-Powell, $\mathrm{MS}^{1}$; Marissa Zwald, PhD ${ }^{2}$; Kayla N. Anderson, PhD²; Rebecca T. Leeb, PhD 3 ; Kristin M. Holland, PhD ${ }^{2}$; Abigail Gates, MSPH'; Jourdan DeVies, MS4; Amanda R. Smith, PhD ${ }^{1,5}$; Katharina L. van Santen, $\mathrm{MSPH}^{4}$; Sophia Crossen, MS ${ }^{6}$; Michael Sheppard, MS ${ }^{1}$; Samantha Wotiz, MPH${ }^{7}$; Amelia G. Johnson, DrPH ${ }^{8}$; Amber Winn, $\mathrm{MPH}^{8}$; Hannah L. Kirking, MD ${ }^{8}$; Rashon I. Lane, PhD, MA ; Rashid Njai, $\mathrm{PhD}^{10}$; Loren Rodgers, $\mathrm{PhD}^{1}$; Craig W. Thomas, PhD ${ }^{11}$; Karl Soetebier, MAPW ${ }^{1}$; Jennifer Adjemian, $\mathrm{PhD}^{1}$
\end{abstract}

On February 18, 2022, this report was posted as an MMWR Early Release on the MMWR website (https://www.cdc.gov/mmwr).

Emergency departments (EDs) in the United States remain a frontline resource for pediatric health care emergencies during the COVID-19 pandemic; however, patterns of health-seeking behavior have changed during the pandemic $(1,2)$. CDC examined changes in U.S. ED visit trends to assess the continued impact of the pandemic on visits among children and adolescents aged $0-17$ years (pediatric ED visits). Compared with 2019, pediatric ED visits declined by $51 \%$ during 2020, 22\% during 2021, and 23\% during January 2022. Although visits for non-COVID-19 respiratory illnesses mostly declined, the proportion of visits for some respiratory conditions increased during January 2022 compared with 2019. Weekly number and proportion of ED visits increased for certain types of injuries (e.g., drug poisonings, self-harm, and firearm injuries) and some chronic diseases, with variation by pandemic year and age group. Visits related to behavioral concerns increased across pandemic years, particularly among older children and adolescents. Health care providers and families should remain vigilant for potential indirect impacts of the COVID-19 pandemic, including health conditions resulting from delayed care, and increasing emotional distress and behavioral health concerns among children and adolescents.

CDC assessed data from the National Syndromic Surveillance Program (NSSP)* for three surveillance periods: March 15, 2020-January 2, 2021 (2020), January 3, 2021January 1, 2022 (2021), and January 2, 2022-January 29, 2022 (January 2022), and compared them with corresponding weeks in 2019 from health care facilities consistently ${ }^{\dagger}$ reporting data during 2019- January 2022. Data were evaluated by total visits among children and adolescents aged $0-17$ years,

\footnotetext{
${ }^{*}$ NSSP is a collaboration among CDC, local, and state health departments, and federal, academic, and private sector partners. https://www.cdc.gov/nssp/index.html

$\dagger$ To reduce artifactual impact from changes in reporting patterns, analyses were restricted to facilities with a coefficient of variation $\leq 40$ and average weekly informative discharge diagnosis $\geq 75 \%$ complete with consistent discharge diagnosis code formatting throughout 2019-2022. Visits from 1,674 facilities from 41 states were eligible to be included in the study. All facilities from three counties in California (El Dorado, Plumas, and Yosemite), the District of Columbia, Florida, Guam, Hawaii, Maryland, Nebraska, Ohio, Oklahoma, South Dakota, Virginia, Wyoming, and one facility from Washington were excluded because they do not meet one of the inclusion criteria.
}

and by three age groups ( $0-4,5-11$, and $12-17$ years), and visit diagnoses. The Healthcare Cost and Utilization Project (HCUP) Clinical Classifications Software Refined (CCSR) (version 2022; CCSR) tool $^{\S}$ was used to group International Classification of Diseases, Tenth Revision, Clinical Modification (ICD-10-CM) codes into clinically meaningful diagnostic categories. All ICD-10-CM codes associated with an ED visit were mapped to CCSR categories using a one-to-many approach: a visit with multiple codes could be counted across more than one category; however, if multiple codes in a single visit mapped to the same category, the visit was counted only once. CDC selected 15 categories with the largest increases and decreases in number of mean weekly visits and identified 20 categories with the largest relative change measured by visit ratios (VRs) and 95\% CIs; CIs that excluded 1 were considered statistically significant. Categories with both largest change in mean weekly visits and in VRs were included only once. To retain practical relevance, categories were restricted to those with a total difference of $\geq 100$ visits between surveillance and comparison periods. All analyses were conducted using $\mathrm{R}$ software (version 4.1.2; R Foundation). This activity was reviewed by CDC and was conducted consistent with applicable federal law and CDC policy.**

Pediatric ED visits declined by $51 \%$ during 2020, 22\% during 2021, and 23\% during January 2022, compared with 2019 (Figure 1), with some differences by age (Figure 2) but negligible differences between sexes. Overall, the largest decreases in mean weekly visits and proportion of visits were for non-COVID-19 respiratory illnesses when compared with

\footnotetext{
$\S$ A full list of categories and corresponding codes is available at the HCUP website: https://www.hcup-us.ahrq.gov/toolssoftware/ccsr/dxccsr.jsp (Accessed January 14, 2022).

9 To assess greatest increases and decreases in mean weekly visits, no filtering on visit count or relative standard error was applied. VRs were calculated as the proportion of all ED visits in each diagnostic category during the pandemic surveillance period, divided by the proportion of all $\mathrm{ED}$ visits in that category during the comparison period. Ninety-five percent CIs that excluded 1 were considered statistically significant. To maintain practical relevance of results, VRs were suppressed if there were $<100$ visits, the difference in visit counts between periods was $<100$, or the relative standard error was $>30 \%$. Ratios of not applicable (N/A) do not meet at least one of those criteria.

** 45 C.F.R. part 46.102(l)(2), 21 C.F.R. part 56; 42 U.S.C. Sect. 241(d); 5 U.S.C. Sect. 552a; 44 U.S.C. Sect. 3501 et seq.
} 


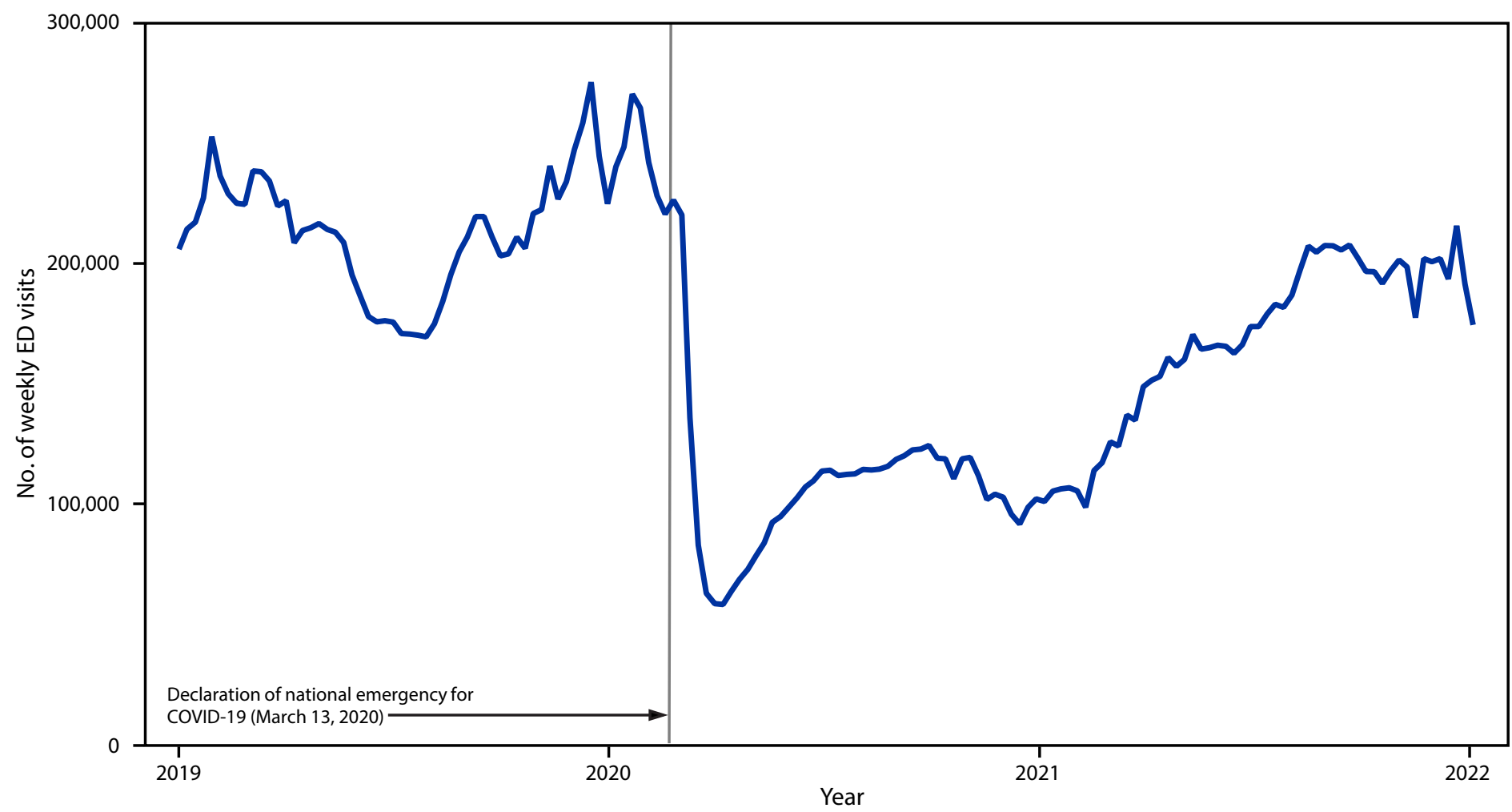

Abbreviations: $\mathrm{ED}=$ emergency department; NSSP = National Syndromic Surveillance Program.

* NSSP is a collaboration among CDC, local, and state health departments, and federal, academic, and private sector partners. To reduce artifactual impact from changes in reporting patterns, analyses were restricted to facilities with a coefficient of variation $\leq 40$ and average weekly informative discharge diagnosis $\geq 75 \%$ complete with consistent discharge diagnosis code formatting throughout 2019-2022. Visits from 1,674 facilities from 41 states were eligible to be included in the study. All facilities from three counties in California (El Dorado, Plumas, and Yosemite), the District of Columbia, Florida, Guam, Hawaii, Maryland, Nebraska, Ohio, Oklahoma, South Dakota, Virginia, Wyoming, and one facility from Washington were excluded because they do not meet one of the inclusion criteria. https://www. cdc.gov/nssp/index.html

2019; however, proportion of visits for fever increased during January 2022 (Supplementary Table 1, https://stacks.cdc. gov/view/cdc/114478). The largest increases in mean weekly visits during 2020-2022 occurred in diagnostic categories for infectious disease exposure and screening, and COVID-19. Proportion of visits also increased for infectious disease exposure and screening. Number and proportion of visits related to pedal cycle injuries and visits for some chronic conditions and their treatment (e.g., specific cancers, cancer therapy, and connective tissue disorders) also increased, with pandemic year and pediatric age variations.

Compared with 2019, the number and proportion of cannabis-involved visits among children aged $0-4$ years increased during 2020 and 2021, with an increase of eight visits per week in $2020(\mathrm{VR}=3.94)$ and an increase of 15 visits per week during 2021 (VR = 3.14). The number and proportion of firearm injury visits among children in this age group also increased during 2020 and 2021, with an increase of three visits per week during $2020(\mathrm{VR}=4.20)$ and two visits per week during 2021
$(\mathrm{VR}=2.33)$. Similarly, the number and proportion of visits for psychosocial factors among children aged 0-4 years increased during 2021 and January 2022, with an increase of 38 visits per week during $2021(\mathrm{VR}=1.53)$ and 55 visits per week during January $2022(\mathrm{VR}=1.91)$ compared with 2019 . The number and proportion of visits for neurodevelopmental disorders among children in this age group also increased during 2021 and January 2022, with an increase of 18 visits per week during $2021(\mathrm{VR}=1.35)$ and 45 visits per week during January 2022 $(\mathrm{VR}=1.71)$ (Supplementary Table 2, https://stacks.cdc.gov/ view/cdc/114478). During January 2022, although visits for epilepsy decreased by 35 visits per week and visits for respiratory symptoms (e.g., cough) decreased by 2,063 visits per week in this age group, the proportion of visits increased both for epilepsy $(\mathrm{VR}=1.37)$ and respiratory symptoms $(\mathrm{VR}=1.12)$.

Among children aged 5-11 years, the number and proportion of cannabis-involved visits increased during 2020 and 2021 compared with 2019, with an increase of four visits per week during $2020(\mathrm{VR}=3.72)$ and an increase of nine visits 
FIGURE 2. Weekly number of emergency department visits* among children and adolescents aged 0-17 years, by age group - National Syndromic Surveillance Program, United States, 2019-2022

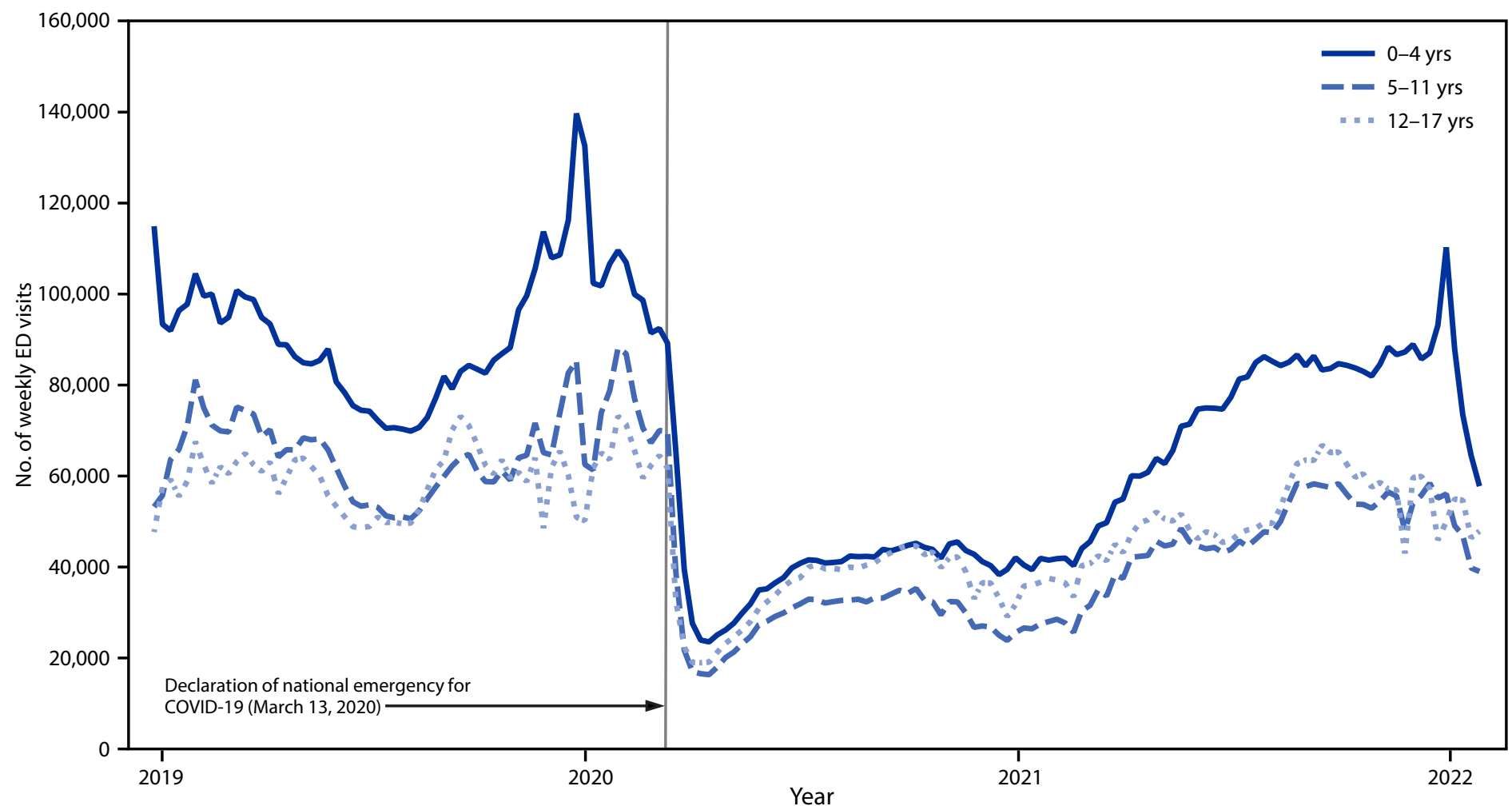

Abbreviations: $E D=$ emergency department; NSSP = National Syndromic Surveillance Program.

* NSSP is a collaboration among CDC, local, and state health departments, and federal, academic, and private sector partners. To reduce artifactual impact from changes in reporting patterns, analyses were restricted to facilities with a coefficient of variation $\leq 40$ and average weekly informative discharge diagnosis $\geq 75 \%$ complete with consistent discharge diagnosis code formatting throughout 2019-2022. Visits from 1,674 facilities from 41 states were eligible to be included in the study. All facilities from three counties in California (El Dorado, Plumas, and Yosemite), the District of Columbia, Florida, Guam, Hawaii, Maryland, Nebraska, Ohio, Oklahoma, South Dakota, Virginia, Wyoming, and one facility from Washington were excluded because they do not meet one of the inclusion criteria. https://www. cdc.gov/nssp/index.html

per week during $2021(\mathrm{VR}=3.54)$ (Supplementary Table 3, https://stacks.cdc.gov/view/cdc/114478). The number and proportion of visits for psychosocial concerns also increased during 2021 and January 2022 compared with 2019, with an increase of 20 visits per week during $2021(\mathrm{VR}=1.74)$ and 35 visits per week during January $2022(\mathrm{VR}=2.18)$. During 2021, the number and proportion of visits for firearm injuries, self-harm, and drug-poisoning were higher compared with 2019, with an increase of two visits per week for firearm injuries $(\mathrm{VR}=2.01)$, six visits per week for self-harm $(\mathrm{VR}=1.69)$, and seven visits per week for drug poisoning $(\mathrm{VR}=1.55)$. During January 2022, although the number of visits for viral infection, fever, and epilepsy decreased compared with 2019, the proportion of visits increased, with a decrease of 286 visits per week for viral infection $(\mathrm{VR}=1.26)$, a decrease of 814 visits per week for fever $(\mathrm{VR}=1.11)$, and a decrease of 38 visits per week for epilepsy $(\mathrm{VR}=1.29)$.
Among adolescents aged 12-17 years, during 2020, 2021, and January 2022, the number and proportion of visits for injuries from physical activities (e.g., walking, swimming, and running) decreased compared with 2019, with decreases of 1,669 visits per week during $2020(\mathrm{VR}=0.80)$, 966 visits per week during $2021(\mathrm{VR}=0.80)$, and 757 visits per week during January $2022(\mathrm{VR}=0.72)$. Conversely, the number and proportion of visits for self-harm increased in all 3 years, with increases of 30 visits per week during 2020 (VR = 1.77), 210 visits per week during $2021(\mathrm{VR}=1.59)$, and 207 visits per week during January $2022(\mathrm{VR}=1.48)$. Similarly, the number and proportion of visits for drug poisonings and eating disorders increased during all 3 years compared with 2019, with increases of 12 visits per week for drug poisonings during 2020 (VR = 1.72), 171 visits per week during 2021 $(\mathrm{VR}=1.53)$, and 178 visits per week during January 2022 $(\mathrm{VR}=1.43)$; and increases of nine visits per week for eating disorders during $2020(\mathrm{VR}=2.00)$, 41 visits per week during 
$2021(\mathrm{VR}=2.14)$, and 38 visits per week during January 2022 $(\mathrm{VR}=1.92)$ (Supplementary Table 4, https://stacks.cdc.gov/ view/cdc/114478). The number and proportion of visits for firearm injuries increased by 22 visits per week $(\mathrm{VR}=2.43)$ during 2020 and by 20 visits per week during January 2022 $(\mathrm{VR}=1.69)$ compared with 2019. The number and proportion of visits for psychosocial concerns and for symptoms of mental health conditions and substance use increased during 2021 and January 2022, with increases of 78 visits per week during $2021(\mathrm{VR}=1.66)$ and 62 visits per week during January $2022(\mathrm{VR}=1.48)$ for psychosocial concerns and increases of 113 visits per week during $2021(\mathrm{VR}=1.35)$ and 197 visits per week during January $2022(\mathrm{VR}=1.43)$ for symptoms of mental conditions and substance use. In January 2022, the number and proportion of visits for respiratory symptoms (e.g., cough) increased by 905 visits per week $(\mathrm{VR}=1.55)$.

\section{Discussion}

Pediatric ED visits sharply declined in the United States during 2020 compared with 2019 (1), and although the weekly numbers of visits have varied, ED visits remained lower during 2021 and January 2022 compared with those before the pandemic (2). These declines might be associated with parents' and caregivers' risk perception and avoidance of EDs or health care, among other reasons. Despite overall declines, weekly ED visits did increase for children aged 0-4 years at the end of 2021, aligning with the increased circulation of the B.1.1.529 (Omicron) variant of SARS-CoV-2, the virus that causes COVID-19, in the United States. These increases were not observed in children aged 5-11 and adolescents aged 12-17 years; both age groups were eligible for vaccination at the end of 2021. COVID-19-associated visits, and those for exposure and screening for infectious disease, were the top two visit diagnoses for children of all age groups during January 2022. Being up to date with vaccinations is critical for adults and eligible children and adolescents ${ }^{\dagger \dagger}$ to prevent infection, severe illness, or death from COVID-19 (3,4), and might reduce strain on health care resources. Supplementary testing $\mathbb{S \$}$ strategies for COVID-19 can further alleviate the impact of the pandemic on EDs $(3,5)$.

\footnotetext{
$\dagger^{\dagger}$ https://www.cdc.gov/coronavirus/2019-ncov/vaccines/recommendations/ children-teens.html (Accessed January 15, 2022). Persons aged 12-17 years became eligible for vaccination on May 10, 2021. https://www.fda.gov/newsevents/press-announcements/coronavirus-covid-19-update-fda-authorizespfizer-biontech-covid-19-vaccine-emergency-use. Children aged 5-11 years became eligible for vaccination on October 29, 2021. Age 5 years is the current youngest age eligible for COVID-19 vaccination. https://www.fda. gov/news-events/press-announcements/fda-authorizes-pfizer-biontech-covid19-vaccine-emergency-use-children-5-through-11-years-age

$\$ \$$ https://www.cdc.gov/coronavirus/2019-ncov/testing/self-testing.html (Accessed January 14, 2022).
}

The proportion of visits for non-COVID-19 respiratory illnesses mostly declined across all periods examined, suggesting that COVID-19 prevention measures might have reduced transmission of other respiratory viruses as well (G). However, during January 2022, the proportion of visits for fever, viral infection, and respiratory symptoms such as cough increased, with variations by age group. Clinicians should remain vigilant for potentially changing clinical COVID-19 presentations associated with the Omicron variant (7), as well as any newly emergent variants of concern.

Elevated proportions of visits for some other diseases, including some chronic conditions and treatments (e.g., cancer therapies), might indicate delay of care and routine well child visits; reduced screening; or postponed procedures to reallocate medical resources during the pandemic $(1,2)$. Health care systems should encourage caregivers of children and adolescents to seek necessary and scheduled care.

Higher numbers and proportions of cannabis-involved visits among children aged $0-11$ years during 2020 and 2021 might be associated with increases in unintentional ingestion. Although there was some variation by pandemic year, increases in visits with certain injuries across all age groups (e.g., firearm injuries), as well as among children and adolescents aged 5-17 years (e.g., drug poisoning and self-harm), are consistent with reports of increased overdose and violence outcomes during the pandemic (8). Factors affecting caregivers, including unavailable or unpredictable child care, illness, financial hardship, and mental health concerns, might increase children and adolescents' vulnerabilities. Children and adolescents' loss of parents or other caregivers (9), increases in other adversities, and disruptions in daily routine because of the COVID-19 pandemic's might also increase children and adolescents' behavioral health concerns and unhealthy coping behaviors. Comprehensive prevention strategies, ${ }^{* *,+\dagger \dagger, \$ \$ \$}$ including strengthening supports to reduce family stress; enhancing access to services and resources; safe storage of firearms and other lethal means; and limiting accessibility to drugs such as cannabis, to reduce use among children and adolescents, can help address these factors. Further, increases in visits for other behavioral concerns and eating disorders align with previous findings, suggesting that the COVID-19 pandemic has exacerbated already high rates of mental health concerns

\footnotetext{
99 https://www.aap.org/en/patient-care/family-snapshot-during-the-covid19-pandemicl (Accessed February 15, 2022).

*** https://www.cdc.gov/violenceprevention/pdf/preventingACES.pdf

${ }^{\dagger \dagger \dagger}$ https://www.cdc.gov/suicide/pdf/suicideTechnicalPackage.pdf. (Accessed January 18, 2022).

$\$ \$ \$ S$ https://www.cdc.gov/violenceprevention/pdf/yv-technicalpackage.pdf (Accessed January 18, 2022).
} 


\section{Summary}

What is already known about this topic?

Health seeking behavior has changed during the COVID-19 pandemic.

What is added by this report?

Compared with 2019, overall pediatric emergency department visits decreased by $51 \%, 22 \%$, and $23 \%$ during 2020, 2021, and January 2022, respectively. COVID-19 visits predominated across all pediatric ages; visits for other respiratory illnesses mostly declined. Number and proportion of visits increased for certain injuries (e.g., firearm injuries, self-harm, and drug poisonings), some chronic diseases, and behavioral health concerns, with variations by age group.

What are the implications for public health practice?

Health care providers and families should remain vigilant for potential indirect impacts of the COVID-19 pandemic, including health conditions resulting from delayed care, and increasing emotional distress and behavioral health concerns among children and adolescents.

among children and adolescents $999, * * * *(10)$. In addition to those who routinely treat children and adolescents' mental and behavioral health, educators and others who work with children and adolescents can also help identify symptoms of distress and unhealthy coping behaviors that might warrant further intervention.

The findings in this report are subject to at least five limitations. First, NSSP ED visit data are a convenience sample and should not be considered nationally representative. Second, fluctuations in underlying data quality, coding practices, and variations in lengths of surveillance periods, particularly during January 2022, might not be reflective of trends from a longer period, potentially overor underrepresenting visit trends. To help account for this, visit data were analyzed only from facilities with consistent reporting during the study period. Third, many factors, including patterns of care-seeking, changed during the COVID-19 pandemic, and this study was not able to draw conclusions about the underlying prevalence of these conditions outside EDs. Fourth, this report assessed trends in ED visits with one or many diagnosis codes; multiple visits by the same patient are possible, and each would be counted separately. Finally, this analysis could not ascertain which diagnosis was the primary reason for the visit; any visit with a relevant diagnosis was included in that clinical category.

Health care systems should be aware of indirect effects of delayed medical care and maintain vigilance for signs of exacerbated

\footnotetext{
999 https://www.aap.org/en/advocacy/child-and-adolescent-healthy-mentaldevelopment/aap-aacap-cha-declaration-of-a-national-emergency-in-childand-adolescent-mental-health/ (Accessed January 14, 2022).

**** https://www.hhs.gov/about/news/2021/12/07/us-surgeon-general-issuesadvisory-on-youth-mental-health-crisis-further-exposed-by-covid-19pandemic.html (Accessed January 14, 2022).
}

emotional distress and behavioral health concerns, especially among older children and adolescents. Prevention programs that improve children and adolescents' physical and mental health are critical during and after emergencies. Reducing COVID-19 infection through vaccination and other nonpharmaceutical prevention strategies can further protect pediatric health.

\section{Acknowledgments}

National Syndromic Surveillance Program Community of Practice, Umed A. Ajani, Noelle-Angelique Molinari, Matthew D. Ritchey, Paula Yoon.

Corresponding author: Lakshmi Radhakrishnan, LRadhakrishnan@cdc.gov.

\begin{abstract}
${ }^{1}$ Division of Health Informatics and Surveillance, Center for Surveillance, Epidemiology, and Laboratory Services, CDC; ${ }^{2}$ Division of Violence Prevention, National Center for Injury Prevention and Control, CDC; ${ }^{3}$ Division of Human Development and Disability, National Center on Birth Defects and Developmental Disabilities, CDC; ${ }^{4}$ ICF International, Fairfax, Virginia; ${ }^{5}$ Epidemic Intelligence Service, CDC; ${ }^{6}$ InductiveHealth Informatics, Atlanta, Georgia; ${ }^{7}$ Deloitte, Atlanta, Georgia; ${ }^{8}$ Division of Viral Diseases, National Center for Immunization and Respiratory Diseases, CDC; ${ }^{9}$ Division of HIV Prevention, National Center for HIV, Viral Hepatitis, STD, and TB Prevention, CDC; ${ }^{10}$ Office of Minority Health and Health Equity, Office of the Deputy Director for Public Health Service and Implementation, CDC; ${ }^{11}$ National Center for Chronic Disease Prevention and Health Promotion, CDC.
\end{abstract}

All authors have completed and submitted the International Committee of Medical Journal Editors form for disclosure of potential conflicts of interest. No potential conflicts of interest were disclosed.

\section{References}

1. Hartnett KP, Kite-Powell A, DeVies J, et al.; National Syndromic Surveillance Program Community of Practice. Impact of the COVID-19 pandemic on emergency department visits-United States, January 1, 2019-May 30, 2020. MMWR Morb Mortal Wkly Rep 2020;69:699-704. PMID:32525856 https://doi.org/10.15585/mmwr.mm6923e1

2. Adjemian J, Hartnett KP, Kite-Powell A, et al. Update: COVID-19 pandemic-associated changes in emergency department visits-United States, December 2020-January 2021. MMWR Morb Mortal Wkly Rep 2021;70:552-6. PMID:33857069 https://doi.org/10.15585/ mmwr.mm7015a3

3. Iuliano $\mathrm{AD}$, Brunkard JM, Boehmer TK, et al. Trends in disease severity and health care utilization during the early Omicron variant period compared with previous SARS-CoV-2 high transmission periodsUnited States, December 2020-January 2022. MMWR Morb Mortal Wkly Rep 2022;71:146-52. PMID:35085225 https://doi.org/10.15585/ mmwr.mm7104e4

4. Olson SM, Newhams MM, Halasa NB, et al.; Overcoming Covid-19 Investigators. Effectiveness of BNT162b2 vaccine against critical Covid-19 in adolescents. N Engl J Med 2022;NEJMoa2117995. PMID:35021004 https://doi.org/10.1056/NEJMoa2117995

5. Nemoto N, Dhillon S, Fink S, et al. Evaluation of test to stay strategy on secondary and tertiary transmission of SARS-CoV-2 in K-12 schools—Lake County, Illinois, August 9-October 29, 2021. MMWR Morb Mortal Wkly Rep 2021;70:1778-81. PMID:34968375 https:// doi.org/10.15585/mmwr.mm705152e2

6. Huang QS, Wood T, Jelley L, et al.; NPIsImpactOnFlu Consortium. Impact of the COVID-19 nonpharmaceutical interventions on influenza and other respiratory viral infections in New Zealand. Nat Commun 2021;12:1001. PMID:33579926 https://doi.org/10.1038/ s41467-021-21157-9 
7. CDC COVID-19 Response Team. SARS-CoV-2 B.1.1.529 (Omicron) Variant - United States, December 1-8, 2021. MMWR Morb Mortal Wkly Rep 2021;70:1731-4. PMID:34914670 https://doi.org/10.15585/ mmwr.mm7050e1

8. Holland KM, Jones C, Vivolo-Kantor AM, et al. Trends in US emergency department visits for mental health, overdose, and violence outcomes before and during the COVID-19 pandemic. JAMA Psychiatry 2021;78:372-9. PMID:33533876 https://doi.org/10.1001/ jamapsychiatry.2020.4402
9. Hillis SD, Blenkinsop A, Villaveces A, et al. COVID-19-associated orphanhood and caregiver death in the United States. Pediatrics 2021;148:e2021053760. PMID:34620728 https://doi.org/10.1542/ peds.2021-053760

10. Whitney DG, Peterson MD. US national and state-level prevalence of mental health disorders and disparities of mental health care use in children. JAMA Pediatr 2019;173:389-91. PMID:30742204 https:// doi.org/10.1001/jamapediatrics.2018.5399 